\title{
RESEARCH
}

Open Access

\section{Liver resection for colorectal liver-limited metastases in elderly patients: a propensity score matching analysis}

Ke-Min Jin, Kun Wang, Quan Bao, Hong-Wei Wang and Bao-Cai Xing*

\begin{abstract}
Background: Few studies have focused on the role of hepatectomy for colorectal liver-limited metastases in elderly patients compared to matched younger patients.

Methods: From January 2000 to December 2018, 724 patients underwent hepatectomy for colorectal liver-limited metastases. Based on a 1:2 propensity score matching (PSM) model, 64 elderly patients ( $\geq 70$ years of age) were matched to 128 younger patients (< 70 years of age) to obtain two balanced groups with regard to demographic, therapeutic, and prognostic factors.

Results: There were 73 elderly and 651 younger patients in the unmatched cohort. Compared with the younger group (YG), the elderly group (EG) had significantly higher proportion of American Society of Anesthesiologists score III and comorbidities and lower proportion of more than 3 liver metastases and postoperative chemotherapy $(p<0.05)$. After PSM for these factors, rat sarcoma virus proto-oncogene/B-Raf proto-oncogene (RAS/BRAF) mutation status and primary tumor sidedness, the EG had significantly less median intraoperative blood loss than the YG (175 ml vs. $200 \mathrm{ml}, p=0.046)$, a shorter median postoperative hospital stay (8 days vs. 11 days, $p=0.020$ ), and a higher readmission rate (4.7\% vs.0\%, $p=0.036)$. The $E G$ also had longer disease-free survival (DFS), overall survival (OS), and cancer-specific survival (CSS) compared to the YG, but these findings were not statistically significant $(p>0.05)$. Old age was not an independent factor for DFS, OS, and CSS by Cox multivariate regression analysis $(p>0.05)$.
\end{abstract}

Conclusions: Hepatectomy is safe for colorectal liver-limited metastases in elderly patients, and these patients may subsequently benefit from prolonged DFS, OS, and CSS.

Keywords: Liver-limited metastases, Colorectal cancer, Elderly, Hepatectomy, Survival

\footnotetext{
* Correspondence: xingbaocai88@sina.com

Key laboratory of Carcinogenesis and Translational Research (Ministry of Education/Beijing), Hepatobiliary and Pancreatic Surgery Unit I, Peking University Cancer Hospital \& Institute, No. 52, Fu Cheng Road, Beijing 100142, People's Republic of China
}

(c) The Author(s). 2020 Open Access This article is licensed under a Creative Commons Attribution 4.0 International License, which permits use, sharing, adaptation, distribution and reproduction in any medium or format, as long as you give appropriate credit to the original author(s) and the source, provide a link to the Creative Commons licence, and indicate if changes were made. The images or other third party material in this article are included in the article's Creative Commons licence, unless indicated otherwise in a credit line to the material. If material is not included in the article's Creative Commons licence and your intended use is not permitted by statutory regulation or exceeds the permitted use, you will need to obtain permission directly from the copyright holder. To view a copy of this licence, visit http://creativecommons.org/licenses/by/4.0/ The Creative Commons Public Domain Dedication waiver (http://creativecommons.org/publicdomain/zero/1.0/) applies to the data made available in this article, unless otherwise stated in a credit line to the data. 


\section{Background}

In 2018, colorectal cancer had the third greatest incidence and the second greatest mortality of all malignant tumors [1]. Approximately half of all colorectal cancer patients have disease that eventually progresses to synchronous or metachronous liver metastases (LM) with or without extrahepatic diseases (EHD) [2, 3]. Radical resection of all liver metastases is the mainstay of management for these patients, which has led to 5-year survival rates of 36 to $58 \%$ [4-9]. However, with the aging of the global population, an increasing number of patients are being diagnosed at an elderly age, when resection may not be a viable treatment option due to a patient's poor performance status or comorbidities [10]. For the elderly patients who do undergo hepatectomy, there have been conflicting results of studies regarding operation safety and long-term survival [11-19]. These studies were not conclusive in part due to biased or missing baseline data, such as comorbidities, the American Society of Anesthesiologists (ASA) score, and preoperative treatment in these studies. To the best of our knowledge, only one study using the propensity score matching method has been published, which demonstrated comparable shortterm and long-term outcomes between the younger group (YG) and the elderly group (EG) [20].

Another flaw of these studies, which investigated the justification for liver resection for colorectal liver metastases in elderly patients, is that they did include patients with EHD. Furthermore, most of the studies did not elaborate on the type and/or management of the EHDs, which could potentially hinder accurate evaluation of disease-free survival. In modern management of colorectal liver metastases, important data such as primary sidedness, RAS/BRAF mutation status, preoperative chemotherapy, and clinical risk score (CRS) were not included in the analyses. The present study was designed to elucidate the role of hepatectomy in elderly patients with colorectal liver-limited metastases using a propensity score matching analysis to overcome the aforementioned limitations of previous work.

\section{Methods}

\section{Data collection}

Data from patients with colorectal liver-limited metastases who underwent complete resection of hepatic metastases between January 2000 and December 2018 at the Hepatobiliary and Pancreatic Surgery Unit I of Beijing Cancer Hospital were retrospectively collected. The study was approved by the hospital's Clinical Research Ethics Committee and was performed in compliance with the Helsinki Declaration. Patients with a second primary malignant tumor were excluded. The bowel and the hepatic resections were performed by a colorectal team and a hepaticobiliary team respectively. For patients with synchronous disease, liver resection was performed if the tumor could be safely resected after assessment of liver remnant reserve and performance status. For those patients with a heavy tumor burden in liver, especially after conversion therapy, liver first approach was adopted usually 2-4 weeks after cessation of preoperative chemotherapy. For those patients with rectal cancer, staged resection was preferred. Otherwise, simultaneous resection was performed. Only the first operation was included for patients who underwent a repeat hepatectomy for disease recurrence. For patients who underwent complete resection by two-stage hepatectomy (portal vein ligation or embolization), only the second surgery-the high-risk right hemi-liver resection-was included. For the purpose of liver parenchyma preservation, we tried to avoid major liver resection $(\geq 3$ segments) for patients with liver metastases. So, when the patient had tumors, which were smaller than $2 \mathrm{~cm}$ in diameter in the deep areas of liver and were not adjacent to large vessels or bile ducts, intraoperative RFA was utilized to reduce the parenchyma loss from otherwise liver resection. All tumor tissues resected prior to 2015 were retrieved and sent for retrospective RAS/BRAF mutation analysis. The patients were followed up every 6 months by hepaticobiliary team by outpatient clinic visit and telephone conversation. The following patient information was evaluated: (1) demographic features, comorbidities and ASA score; (2) primary tumor sidedness, $\mathrm{T}$ stage and $\mathrm{N}$ stage; (3) number, distribution, and maximum diameter of liver metastases; (4) preoperative serum levels of the tumor marker carcinoembryonic antigen (CEA) and carbohydrate antigen 19-9 (CA19-9), temporal relationship of primary tumor and liver metastases, preoperative CRS, preoperative chemotherapy, and RAS/BRAF mutation status; (5) operation time, procedural details (major hepatectomy, combination with radiofrequency ablation (RFA), simultaneous resection, Pringle clamping time of the hepaticoduodenal ligament), intraoperative blood loss, and red blood cell (RBC) transfusion and margin status; (6) postoperative hospital stay and postoperative general and surgical complications; (7) postoperative adjuvant chemotherapy protocol and history of repeat hepatectomy after recurrence; and (8) postoperative followup records of recurrence and death.

\section{Study population}

All patients who underwent complete resection of colorectal liver-limited metastases with confirmed pathologic diagnosis were enrolled in this study. Seventy years of age was defined as the minimum age for elderly patients. Thus, the patients are divided into two groups-the YG ( $<70$ years of age) and the EG ( $\geq 70$ years of age).

\section{Statistical analysis}

Categorical variables were expressed as proportions and numerical variables were expressed as median and range. 
Categorical variables were compared by the chi-square or Fisher's exact tests as appropriate whereas numerical variables were compared using the Mann-Whitney $U$ test. The linear correlation coefficient was used to assess a potential relationship between two numerical variables. To compensate for the biases between the YG and the EG in the unmatched cohort, the propensity score (PS) "nearest neighbor" matching method was used with a matching ratio of 1:2 for the EG and the YG. The caliper value was set at 0.05 . The standardized mean difference was used to assess the imbalance before and after PS matching. The following variables were included in the PS matching model: gender, ASA score, comorbidities, primary $\mathrm{N}$ stage, number of liver metastases, preoperative CEA, preoperative CRS score, RAS/BRAF mutation status, preoperative chemotherapy cycles, history of major liver resection, history of hepatectomy combined with intraoperative RFA, history of repeat hepatectomy after recurrence, and post-hepatectomy adjuvant chemotherapy. Response to preoperative chemotherapy was not included in the PS matching model due to too much missing data. Short-term results such as operation time, intraoperative blood loss, intraoperative and postoperative RBC transfusion, postoperative hospital stay, ICU stay, and Clavien-Dindo grades of general or surgical complications were compared between the EG and the YG before and after PS matching. Recurrence and survival data were followed up by telephone or outpatient visit every 6 months following hepatectomy. Disease-free survival (DFS) was defined as the duration from the date of hepatectomy to the date of event (tumor recurrence or death) or the last follow-up. Overall survival (OS) was defined as the duration from the date of hepatectomy to the date of death or the last follow-up. Cancer-specific survival (CSS) was defined as the duration from the date of hepatectomy to the date of cancer-related death or the last follow-up. Kaplan-Meier survival analysis was used to compare the DFS, OS, and CSS before and after PS matching by the log rank test. The Cox multivariate proportional hazards model was used to identify independent prognostic factors of DFS, OS, and CSS after PS matching. $P<0.05$ was deemed significantly different. All statistical analyses were performed using an R-based extension bundle on the SPSS software package (version 23, SPSS Inc., Chicago, IL, USA).

\section{Results}

\section{Comparison of elderly and younger patients before PS matching}

\section{Demographic characteristics and short-term outcomes}

A total of 724 patients with colorectal liver-limited metastases were included in the study, with 73 patients in the EG and 651 patients in the YG. As shown in Tables 1 and 2, the median age was 56 years old for the YG and 73 years old for the EG $(p<0.001)$. There were 55 patients $(8.4 \%)$ in the YG and 25 patients (34.2\%) in the EG with an ASA score of III $(p<0.001)$. There were 274 patients $(42.1 \%)$ in the YG and 50 patients (68.5\%) in the EG with associated comorbidities $(p<0.001)$. With regard to specific comorbidities, there was a significantly higher percentage of patients with hypertension and ischemic heart disease in the EG than in the YG $(p<0.001$ and $p=0.036$, respectively). A significantly greater proportion of patients had more than 3 liver metastases $(31.3 \%$ vs. $19.2 \%, p=0.032)$ or received more than six cycles of chemotherapy $(13.2 \%$ vs $4.1 \%, p=$ $0.025)$ in the YG compared to the EG. In addition, a greater proportion of patients received postoperative adjuvant chemotherapy in the YG compared to the EG (67.9\% versus $54.8 \%, p=0.024)$. With regard to intraoperative information, a higher proportion of patients in the YG received combined hepatectomy and RFA compared to the EG $(13.1 \%$ vs. $4.1 \%, p=0.017)$. Regarding postoperative outcomes, the Clavien-Dindo grades of both general and surgical complications did not significantly differ between groups, nor did in-hospital or 90-day mortality $(p>0.05)$. However, significantly greater proportions of patients in the EG were admitted to the ICU or readmitted to the hospital postoperatively compared to the YG $(11.0 \%$ vs. $2.8 \%, p=$ 0.003 and $4.1 \%$ vs. $0.5 \%, p=0.016$, respectively).

\section{Long-term outcomes}

The median follow-up period was 28.4 months. The recurrence rates-including both intrahepatic and extrahepatic recurrence-were not significantly different between the EG and YG $(p>0.05)$. There was also no significant difference in 1-year, 3-year, or 5-year DFS, OS, or CSS survival rates ( $p>0.05$; Table 2 and Fig. 1a-c).

\section{Comparison of elderly and younger patients after PS matching \\ Demographic characteristics and short-term outcomes}

After PS matching for preoperative and prognostic factors that differed significantly between groups, a total of 64 cases from the EG and 128 cases from the YG were considered for matched analysis. As shown in Tables 3 and 4, the median ages of the EG and YG groups were 72.5 and 57 years old, respectively $(p<0.001)$. When the biases associated with differences in ASA score, comorbidities, number of liver metastases, number of preoperative chemotherapy cycles, intraoperative RFA, and postoperative adjuvant chemotherapy were removed by PS matching, the following intraoperative and postoperative differences between the groups were found. First, the median intraoperative blood loss in the YG was significantly higher than that in the EG $(200 \mathrm{~mL}$ vs $175 \mathrm{~mL}, p=0.046)$, and the median postoperative hospital stay was significantly longer in the YG than the EG ( 11 days vs. 8 days, $p=0.020$ ). However, the readmission rate of the EG was 
Table 1 Demographics comparison of the elderly and younger patients before PS matching

\begin{tabular}{|c|c|c|c|c|}
\hline Variable & Aged $<70$ years $(n=651)$ & Aged $\geq 70$ years $(n=73)$ & $P$ value & Standardized mean difference \\
\hline Age (years) & $56(19-69)$ & $73(70-83)$ & $<0.001$ & \\
\hline Gender, male & $417(64.1 \%)$ & $51(69.9 \%)$ & 0.325 & 0.173 \\
\hline ASA & & & $<0.001$ & 0.487 \\
\hline$|-| \mid$ & $596(91.6 \%)$ & $48(65.8 \%)$ & & \\
\hline III-IV & $55(8.4 \%)$ & $25(34.2 \%)$ & & \\
\hline Comorbidity & $274(42.1 \%)$ & $50(68.5 \%)$ & $<0.001$ & 0.564 \\
\hline Cerebrovascular disease & $17(2.6 \%)$ & $5(6.8 \%)$ & 0.061 & 0.134 \\
\hline Arrhythmia & $9(1.4 \%)$ & $1(1.4 \%)$ & 1.000 & $<0.001$ \\
\hline Ischemic heart disease & $36(5.5 \%)$ & $9(12.3 \%)$ & 0.036 & 0.474 \\
\hline Diabetes mellitus & $94(14.4 \%)$ & $15(20.5 \%)$ & 0.166 & 0.265 \\
\hline Hypertension & $178(27.3 \%)$ & $40(54.8 \%)$ & $<0.001$ & 0.648 \\
\hline Chronic obstructive pulmonary disease & $5(0.8 \%)$ & $2(2.7 \%)$ & 0.151 & 0.362 \\
\hline Chronic renal dysfunction & $4(0.6 \%)$ & $1(1.4 \%)$ & 0.413 & 0.242 \\
\hline Accompanying liver disease & $29(4.5 \%)$ & $2(2.7 \%)$ & 0.760 & 0.198 \\
\hline Primary tumor sideness & & & 0.198 & \\
\hline Right side & $112(17.2 \%)$ & $17(23.3 \%)$ & & \\
\hline Left side & $539(82.8 \%)$ & $56(76.7 \%)$ & & \\
\hline Primary $\top^{\top}$ & & & 0.659 & \\
\hline pT1-T2 & $64(9.8 \%)$ & $6(8.2 \%)$ & & \\
\hline pT3-T4 & $587(90.2 \%)$ & $67(91.8 \%)$ & & \\
\hline Primary N & & & 0.869 & 0.087 \\
\hline pNO & $199(30.6 \%)$ & $23(31.5 \%)$ & & \\
\hline $\mathrm{pN1} 1-2$ & $452(69.4 \%)$ & $50(68.5 \%)$ & & \\
\hline Number of liver metastases(LM) & & & 0.032 & 0.674 \\
\hline$\leq 3$ & $447(68.7 \%)$ & $59(80.8 \%)$ & & \\
\hline$>3$ & $204(31.3 \%)$ & $14(19.2 \%)$ & & \\
\hline Distribution of LM & & & 0.084 & \\
\hline Unilobar & $341(52.4 \%)$ & $46(63.0 \%)$ & & \\
\hline Bilobar & $310(47.6 \%)$ & $27(37.0 \%)$ & & \\
\hline Maximum diameter of $L M$ & & & 0.631 & \\
\hline$\leq 5 \mathrm{~cm}$ & $566(86.9 \%)$ & $62(84.9 \%)$ & & \\
\hline$>5 \mathrm{~cm}$ & $85(13.1 \%)$ & $11(15.1 \%)$ & & \\
\hline Temporal relationship & & & 0.796 & \\
\hline Synchronous & $367(56.4 \%)$ & $40(54.8 \%)$ & & \\
\hline Metachronous & $284(44.6 \%)$ & $33(45.2 \%)$ & & \\
\hline Preoperative chemotherpy cycles & & & 0.025 & 0.743 \\
\hline$\leq 6$ cycles & $565(86.8 \%)$ & 70 (95.9\%) & & \\
\hline$>6$ cycles & $86(13.2 \%)$ & $3(4.1 \%)$ & & \\
\hline Preoperative clinical risk score (CRS) & & & 0.590 & 0.234 \\
\hline $0-2$ & $344(52.8 \%)$ & $41(56.2 \%)$ & & \\
\hline $3-5$ & $307(47.2 \%)$ & $32(43.8 \%)$ & & \\
\hline RAS/BRAF mutation & 215(33.0\%) & $27(37.0 \%)$ & 0.496 & 0.286 \\
\hline Preoperative CEA (ng/ml) & $8.19(0.47-1351.00)$ & $8.53(1.23-224.80)$ & 0.566 & 0.344 \\
\hline Preoperative CA199(U/ml) & $22.03(0.00-29909.00)$ & $24.56(0.00-1354.00)$ & 0.633 & \\
\hline Repeat resection after recurrence & $73(11.2 \%)$ & $8(11.0 \%)$ & 0.948 & 0.063 \\
\hline Postoperative chemotherapy & $442(67.9 \%)$ & $40(54.8 \%)$ & 0.024 & 0.766 \\
\hline
\end{tabular}


Table 2 Comparison of intraoperative factors and postoperative short-term and long-term results before PS matching

\begin{tabular}{|c|c|c|c|c|}
\hline Variable & Aged $<70$ years $(n=651)$ & Aged $\geq 70$ years $(n=73)$ & $P$ value & Standardized mean difference \\
\hline Combined with RFA & $85(13.1 \%)$ & $3(4.1 \%)$ & 0.027 & 0.486 \\
\hline Two-stage hepatectomy & $9(1.4 \%)$ & $1(1.4 \%)$ & 1.000 & \\
\hline Simultaneous resection & $120(18.4 \%)$ & $17(23.3 \%)$ & 0.315 & \\
\hline Major hepatectomy & $127(19.5 \%)$ & $9(12.3 \%)$ & 1.136 & 0.127 \\
\hline Pringle clamp & $513(78.8 \%)$ & $53(72.6 \%)$ & 0.224 & \\
\hline Pringle clamp time (min) & $20(0-98)$ & $15(0-60)$ & 0.061 & \\
\hline R1 margin & $96(14.7 \%)$ & $7(9.6 \%)$ & 0.232 & \\
\hline Intraoperative blood loss (ml) & $200(0-6500)$ & $150(20-1000)$ & 0.529 & \\
\hline Intraoperative RBC transfusion & $28(4.3 \%)$ & $5(6.8 \%)$ & 0.367 & \\
\hline Intraoperative RBC transfused (U) & $4(1-12)$ & $3(2-4)$ & 0.269 & \\
\hline Operation time (min) & $187(32-600)$ & $180(60-330)$ & 0.146 & \\
\hline Hospital stay (days) & $9(4-78)$ & $9(4-48)$ & 0.909 & \\
\hline Postoperative complications & $186(28.6 \%)$ & $20(27.4 \%)$ & 0.833 & \\
\hline Clavien-Dindo classification & & & 0.262 & \\
\hline$|-| \mid$ & $106(57.0 \%)$ & $14(70.0 \%)$ & & \\
\hline||$I-V$ & $80(43.0 \%)$ & $6(30.0 \%)$ & & \\
\hline General complications & $58(8.9 \%)$ & $4(5.5 \%)$ & 0.321 & \\
\hline Postoperative heart failure & $2(0.3 \%)$ & $0(0 \%)$ & 1.000 & \\
\hline Postoperative coronary artery disease & $6(0.9 \%)$ & $0(0 \%)$ & 1.000 & \\
\hline Postoperative arrhythmia & $8(1.2 \%)$ & $1(1.4 \%)$ & 1.000 & \\
\hline Postoperative lung infection & $5(0.8 \%)$ & $1(1.4 \%)$ & 0.473 & \\
\hline Postoperative renal failure & $2(0.3 \%)$ & $0(0 \%)$ & 1.000 & \\
\hline Postoperative pulmonary embolism & $1(0.2 \%)$ & $0(0 \%)$ & 1.000 & \\
\hline Postoperative deep vein thrombosis & $2(0.3 \%)$ & $0(0 \%)$ & 1.000 & \\
\hline Postoperative urinary infection & $4(0.6 \%)$ & $0(0 \%)$ & 1.000 & \\
\hline Postoperative pleural effusion & $28(4.3 \%)$ & $2(2.7 \%)$ & 0.759 & \\
\hline Postoperative stress ulcer & $6(0.9 \%)$ & $1(1.4 \%)$ & 0.526 & \\
\hline Surgical complications & $129(19.8 \%)$ & $11(15.1 \%)$ & 0.330 & \\
\hline Posthepatectomy liver failure & $22(3.4 \%)$ & $2(2.7 \%)$ & 1.000 & \\
\hline Postoperative abdominal infection & $53(8.1 \%)$ & $7(9.6 \%)$ & 0.671 & \\
\hline Postoperative bile leakage & $37(5.7 \%)$ & $3(4.1 \%)$ & 0.788 & \\
\hline Postoperative abdominal collection & $22(3.4 \%)$ & $2(2.7 \%)$ & 1.000 & \\
\hline Incision infection & $9(1.4 \%)$ & $0(0 \%)$ & 0.610 & \\
\hline Postoperative ileus & $12(1.8 \%)$ & $0(0 \%)$ & 0.622 & \\
\hline Postoperative abdominal bleeding & $26(4.0 \%)$ & $1(1.4 \%)$ & 0.508 & \\
\hline ICU & $18(2.8 \%)$ & $8(11.0 \%)$ & 0.003 & \\
\hline ICU stay (days) & $1(1-6)$ & $1.5(1-6)$ & 0.927 & \\
\hline Postoperative RBC transfusion & $50(7.7 \%)$ & $7(9.6 \%)$ & 0.566 & \\
\hline Postoperative RBC transfused $(U)$ & $4(2-58)$ & $2(2-6)$ & 0.510 & \\
\hline Reoperation & $12(1.8 \%)$ & $0(0 \%)$ & 0.622 & \\
\hline Readmission & $3(0.5 \%)$ & $3(4.1 \%)$ & 0.016 & \\
\hline Mortality (in-hospital) & $1(0.2 \%)$ & $0(0 \%)$ & 1.000 & \\
\hline Mortality (90-day) & $1(0.2 \%)$ & $0(0 \%)$ & 1.000 & \\
\hline Recurrence & $467(71.7 \%)$ & $49(67.1 \%)$ & 0.409 & \\
\hline
\end{tabular}


Table 2 Comparison of intraoperative factors and postoperative short-term and long-term results before PS matching (Continued)

\begin{tabular}{|c|c|c|c|c|}
\hline Variable & Aged $<70$ years $(n=651)$ & Aged $\geq 70$ years $(n=73)$ & $P$ value & Standardized mean difference \\
\hline Intrahepatic recurrence & $355(54.5 \%)$ & $40(54.8 \%)$ & 0.966 & \\
\hline Extrahepatic recurrence & $112(17.2 \%)$ & $9(12.3 \%)$ & 0.290 & \\
\hline Disease-free survival & & & 0.374 & \\
\hline 1-year & $46.6 \%$ & $50.5 \%$ & & \\
\hline 3-year & $26.2 \%$ & $31.0 \%$ & & \\
\hline 5-year & $23.5 \%$ & $25.5 \%$ & & \\
\hline Overall survival & & & 0.219 & \\
\hline 1-year & $94.1 \%$ & $90.4 \%$ & & \\
\hline 3-year & $60.5 \%$ & $56.3 \%$ & & \\
\hline 5-year & $48.7 \%$ & $43.6 \%$ & & \\
\hline Cancer-specific survival & & & 0.512 & \\
\hline 1-year & $94.4 \%$ & $90.4 \%$ & & \\
\hline 3-year & $61.1 \%$ & $59.0 \%$ & & \\
\hline 5-year & $49.2 \%$ & $45.7 \%$ & & \\
\hline
\end{tabular}
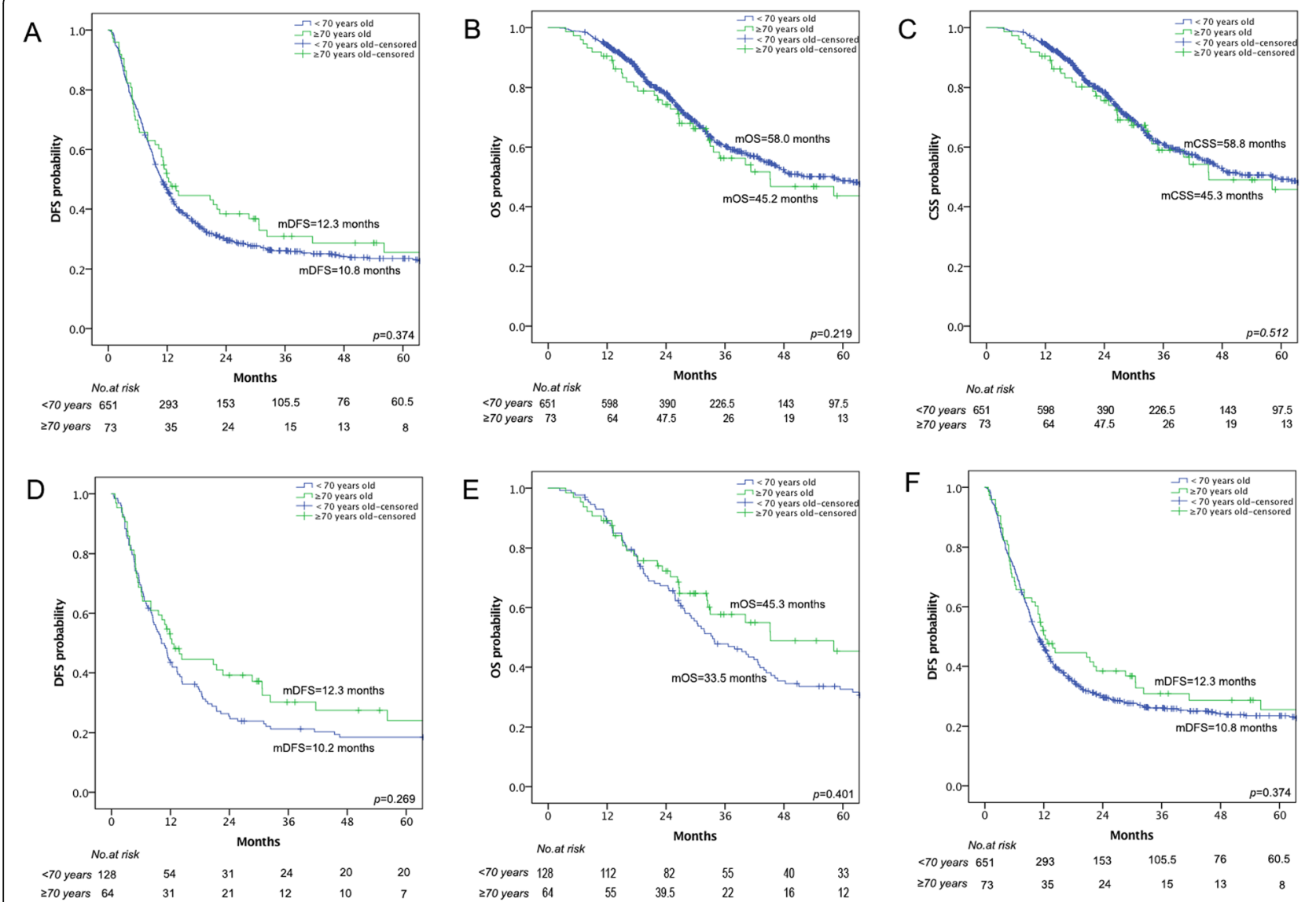

Fig. 1 Comparison of disease-free survial (DFS), overall survival (OS), and cancer-specific survival (CSS) between the elderly group (EG) and the younger group (YG) before (a-c) and after propensity score matching $(\mathbf{d}-\mathbf{f})$ 
Table 3 Demographics comparison of the elderly and younger patients after PS matching

\begin{tabular}{|c|c|c|c|c|}
\hline Variable & Aged $<70$ years $(n=128)$ & Aged $\geq 70$ years $(n=64)$ & $P$ value & Standardized mean difference \\
\hline Age (years) & $57(31-69)$ & $72.5(70-83)$ & $<0.001$ & \\
\hline Gender, male & $73(57.0 \%)$ & $43(67.2 \%)$ & 0.175 & 0.075 \\
\hline ASA & & & 0.200 & 0.083 \\
\hline$|-| \mid$ & $106(82.8 \%)$ & $48(75.0 \%)$ & & \\
\hline III & $22(17.2 \%)$ & $16(25.0 \%)$ & & \\
\hline Comorbidity & $65(50.8 \%)$ & $41(61.1 \%)$ & 0.081 & 0.086 \\
\hline Cerebrovascular disease & $4(3.1 \%)$ & $2(3.1 \%)$ & 1.000 & $<0.001$ \\
\hline Arrhythmia & $3(2.3 \%)$ & $1(1.6 \%)$ & 1.000 & $<0.001$ \\
\hline Ischemic heart disease & $11(8.6 \%)$ & $6(9.4 \%)$ & 0.857 & 0.076 \\
\hline Diabetes mellitus & $21(16.4 \%)$ & $12(18.8 \%)$ & 0.685 & 0.089 \\
\hline Hypertension & $49(38.3 \%)$ & $31(48.4 \%)$ & 0.178 & 0.077 \\
\hline Chronic obstructive pulmonary disease & $0(0.0 \%)$ & $1(1.6 \%)$ & 0.333 & 0.083 \\
\hline Chronic renal dysfunction & $0(0.0 \%)$ & $1(1.6 \%)$ & 0.333 & 0.065 \\
\hline Accompanying liver disease & $10(7.8 \%)$ & $2(3.1 \%)$ & 0.343 & 0.076 \\
\hline Primary tumor sideness & & & 0.447 & \\
\hline Right side & $24(18.8 \%)$ & $15(23.4 \%)$ & & \\
\hline Left side & $104(81.3 \%)$ & $49(76.6 \%)$ & & \\
\hline Primary $T$ & & & 0.557 & \\
\hline pT1-T2 & $8(6.3 \%)$ & $6(9.4 \%)$ & & \\
\hline pT3-T4 & $120(93.8 \%)$ & $58(90.6 \%)$ & & \\
\hline Primary N & & & 0.653 & 0.035 \\
\hline pNO & $36(28.1 \%)$ & $20(31.3 \%)$ & & \\
\hline $\mathrm{pN} 1-2$ & $92(71.9 \%)$ & $44(68.8 \%)$ & & \\
\hline Number of liver metastases $(\mathrm{LM})$ & & & 0.433 & 0.056 \\
\hline$\leq 3$ & $106(82.8 \%)$ & $50(78.1 \%)$ & & \\
\hline$>3$ & $22(17.2 \%)$ & $14(21.9 \%)$ & & \\
\hline Distribution of LM & & & 1.000 & \\
\hline Unilobar & $80(62.5 \%)$ & $40(62.5 \%)$ & & \\
\hline Bilobar & $48(37.5 \%)$ & $24(37.5 \%)$ & & \\
\hline Maximum diameter of LM & & & 0.509 & \\
\hline$\leq 5 \mathrm{~cm}$ & $103(80.5 \%)$ & $54(84.4 \%)$ & & \\
\hline$>5 \mathrm{~cm}$ & $25(19.5 \%)$ & $10(15.6 \%)$ & & \\
\hline Temporal relationship & & & 0.126 & \\
\hline Synchronous & $61(47.7 \%)$ & $38(59.4 \%)$ & & \\
\hline Metachronous & $67(52.3 \%)$ & $26(40.6 \%)$ & & \\
\hline Preoperative chemotherpy cycles & & & 1.000 & $<0.001$ \\
\hline$\leq 6$ cycles & $122(95.3 \%)$ & $61(95.3 \%)$ & & \\
\hline$>6$ cycles & $6(4.7 \%)$ & $3(4.7 \%)$ & & \\
\hline Preoperative clinical risk score (CRS) & & & 0.535 & 0.065 \\
\hline $0-2$ & $76(59.4 \%)$ & $35(54.7 \%)$ & & \\
\hline $3-5$ & $52(40.6 \%)$ & $29(45.3 \%)$ & & \\
\hline RAS/BRAF mutation & $47(36.9 \%)$ & $22(34.4 \%)$ & 0.750 & 0.046 \\
\hline Preoperative CEA (ng/ml) & $9.10(0.93-794.50)$ & $9.41(1.23-224.80)$ & 0.858 & 0.102 \\
\hline Preoperative CA199 (U/ml) & $28.11(0.00-28,385.00)$ & $24.06(0.00-1354.00)$ & 0.360 & \\
\hline
\end{tabular}


Table 3 Demographics comparison of the elderly and younger patients after PS matching (Continued)

\begin{tabular}{lllll}
\hline Variable & Aged $<\mathbf{7 0}$ years $(\boldsymbol{n}=\mathbf{1 2 8})$ & Aged $\geq \mathbf{7 0}$ years $(\boldsymbol{n}=\mathbf{6 4})$ & $\boldsymbol{P}$ value & Standardized mean difference \\
\hline Repeat resection after recurrence & $20(15.6 \%)$ & $8(12.5 \%)$ & 0.563 & 0.078 \\
Postoperative chemotherapy & $86(67.2 \%)$ & $38(59.4 \%)$ & 0.286 & 0.094 \\
$\begin{array}{l}\text { Preoperative chemotherapy regimes } \\
\text { Oxaliplatin-based }\end{array}$ & $37(28.9 \%)$ & $29(45.3 \%)$ & 0.063 \\
Irinotecan-based & $8(6.3 \%)$ & $5(7.8 \%)$ & \\
5-Fu-based & $1(0.8 \%)$ & $2(3.1 \%)$ & \\
Hepatic artery infusion & $4(3.1 \%)$ & $1(1.6 \%)$ & \\
Postoperative chemotherapy regimes & & & \\
Oxaliplatin-based & $50(39.1 \%)$ & $28(43.8 \%)$ & \\
Irinotecan-based & $22(17.2 \%)$ & $6(9.4 \%)$ & \\
5-Fu-based & $8(6.3 \%)$ & $3(4.7 \%)$ & \\
Hepatic artery infusion & $1(0.8 \%)$ & $0(0 \%)$ & \\
\hline
\end{tabular}

still significantly greater than that of YG $(4.7 \%$ vs. $0.0 \%$, $p=0.036$ ). Other postoperative variables, such as ClavienDindo grades for surgical and general complications, postoperative ICU stay, postoperative RBC transfusion, and reoperation rate, were not significantly different between groups $(p>0.05)$. In addition, the in-hospital and 90-day mortality rates were similar between the two groups $(p>$ 0.05).

\section{Long-term outcomes}

The median follow-up period for the matched patient groups was 29.8 months. The recurrence rates-including both intra- and extra-hepatic recurrence-between the EG and YG were not significantly different $(p>$ 0.05). The 1-year, 3-year, and 5-year DFS, OS, and CSS survival rates were higher in the EG than in the YG; however, this difference was not statistically significant $(p>0.05$; Table 4 and Fig. $1 \mathrm{~d}-\mathrm{f})$. In the EG, the 5-year DFS, OS, and CSS rates were $24.1 \%, 45.4 \%$, and $48.0 \%$, respectively; the median lengths of DFS, OS, and CSS were 12.3 months, 45.3 months, and 58.2 months respectively. In the YG, the 5 -year DFS, OS, and CSS rates were $18.5 \%, 32.7 \%$, and $32.9 \%$ respectively; the median lengths of DFS, OS, and CSS were 10.2 months, 33.5 months, and 33.5 months respectively.

\section{Cox proportional hazards model analysis}

Cox multivariate regression analysis was performed for the PS-matched cohort, which included 64 EG patients and 128 YG patients. As shown in Table 5, RAS/BRAF mutation status, preoperative serum CEA levels $\geq 20 \mathrm{ng} / \mathrm{mL}$, preoperative CRS $\geq 3$, and the presence of $>3$ liver metastases were identified as independent predictive factors of DFS $(p<0.05)$. Preoperative serum CEA levels $\geq 20 \mathrm{ng} / \mathrm{mL}$ and preoperative CRS $\geq 3$ were identified as independent predictive factors of both OS and CSS $(p<0.05)$. Notably, old age ( $\geq 70$ years) was not identified as an independent predictive factor for DFS, OS, or CSS $(p>0.05)$.

\section{Discussion}

With the increasing age of the global population, stage IV colorectal cancer is being diagnosed in elderly patients more frequently than ever before [10]. As a result of extensive progress in surgical and anesthetic techniques and modern chemotherapy regimens, more patients can undergo resection of liver metastases with curative intent. This has been proven to be the most effective treatment strategy for colorectal liver metastases (CRLM). However, the increasing possibility of age-related comorbidities and high ASA scores among the elderly patients undergoing hepatectomy poses a higher risk of postoperative morbidity and mortality. Most recently, a multidisciplinary consensus from Italy pointed out the potentially negative impact of old age on recovery of patients from damage. The consensus also put forward some useful evaluation benchmarks for the elderly before operation, which would be critical for the selection of the elderly patients for surgery in the futur e[21]. Although some previous literature reported that older patients had similar surgical safety and long-term survival compared with younger patients, baseline data for both groups were not balanced. Some important prognostic factors such as RAS/BRAF mutation and primary tumor sidedness were also not included in published studies. Therefore, this study, which compared the short-term and long-term results of hepatectomy for younger and elderly patients, was designed to overcome the abovementioned drawbacks.

There are different definitions in the literature as to the cutoff age for an individual to be designated as elderly $[11,15-18,20]$. However, the most frequently used cutoff age is $70[15,16,22-24]$, which was adopted for the EG cutoff in this study. Due to the unmatched demographic and preoperative treatment data between 
Table 4 Comparison of intraoperative factors and postoperative short-term and long-term results after PS matching

\begin{tabular}{|c|c|c|c|c|}
\hline Variable & Aged $<70$ years $(n=128)$ & Aged $\geq 70$ years $(n=64)$ & $P$ value & Standardized mean difference \\
\hline Combined with RFA & $1(0.8 \%)$ & $3(4.7 \%)$ & 0.109 & 0.043 \\
\hline Two-stage hepatectomy & $1(0.8 \%)$ & $1(1.6 \%)$ & 1.000 & \\
\hline Simultaneous resection & $17(13.3 \%)$ & $16(25.0 \%)$ & 0.066 & \\
\hline Major hepatectomy & $24(18.8 \%)$ & $8(12.5 \%)$ & 0.273 & 0.023 \\
\hline Pringle clamp & $95(74.2 \%)$ & $44(68.8 \%)$ & 0.424 & \\
\hline Pringle clamp time (min) & $15(0-60)$ & $15(0-60)$ & 0.465 & \\
\hline R1 margin & $3(2.3 \%)$ & $6(9.4 \%)$ & 0.062 & \\
\hline Intraoperative blood loss (ml) & $200(20-6500)$ & $175(20-800)$ & 0.046 & \\
\hline Intraoperative RBC transfusion & $10(7.9 \%)$ & $3(4.7 \%)$ & 0.549 & \\
\hline Intraoperative RBC transfused (U) & $0(0-8)$ & $0(0-4)$ & 0.313 & \\
\hline Operation time (min) & $172.5(60-570)$ & $180(60-327)$ & 0.799 & \\
\hline Hospital stay (days) & $11(4-70)$ & $8(4-48)$ & 0.020 & \\
\hline Postoperative complications & $37(28.9 \%)$ & $17(26.6 \%)$ & 0.733 & \\
\hline Clavien-Dindo classification & & & 0.062 & \\
\hline$|-| \mid$ & $16(43.2 \%)$ & $12(70.6 \%)$ & & \\
\hline||$I-V$ & $21(56.8 \%)$ & $5(29.4 \%)$ & & \\
\hline General complications & $15(11.7 \%)$ & $4(6.3 \%)$ & 0.232 & \\
\hline Postoperative heart failure & $0(0.0 \%)$ & $0(0 \%)$ & - & \\
\hline Postoperative coronary artery disease & $2(1.6 \%)$ & $0(0 \%)$ & 0.553 & \\
\hline Postoperative arrhythmia & $0(0.0 \%)$ & $1(1.6 \%)$ & 0.333 & \\
\hline Postoperative lung infection & $1(0.8 \%)$ & $1(1.6 \%)$ & 1.000 & \\
\hline Postoperative renal failure & $0(0.0 \%)$ & $0(0.0 \%)$ & - & \\
\hline Postoperative pulmonary embolism & $1(0.8 \%)$ & $0(0.0 \%)$ & 1.000 & \\
\hline Postoperative deep vein thrombosis & $0(0.0 \%)$ & $0(0.0 \%)$ & - & \\
\hline Postoperative urinary infection & $0(0.0 \%)$ & $0(0.0 \%)$ & - & \\
\hline Postoperative pleural effusion & $10(7.8 \%)$ & $2(3.1 \%)$ & 0.343 & \\
\hline Postoperative stress ulcer & $1(0.8 \%)$ & $1(1.6 \%)$ & 1.000 & \\
\hline Surgical complications & $24(18.8 \%)$ & $8(12.5 \%)$ & 0.273 & \\
\hline Posthepatectomy liver failure & $2(1.6 \%)$ & $1(1.6 \%)$ & 1.000 & \\
\hline Postoperative abdominal infection & $3(2.3 \%)$ & $5(7.8 \%)$ & 0.120 & \\
\hline Postoperative bile leakage & $8(6.3 \%)$ & $2(3.1 \%)$ & 0.500 & \\
\hline Postoperative abdominal collection & $4(3.1 \%)$ & $2(3.1 \%)$ & 1.000 & \\
\hline Incision infection & $3(2.3 \%)$ & $0(0.0 \%)$ & 0.552 & \\
\hline Postoperative ileus & $4(3.1 \%)$ & $0(0.0 \%)$ & 0.303 & \\
\hline Postoperative abdominal bleeding & $5(3.9 \%)$ & $1(1.6 \%)$ & 0.666 & \\
\hline ICU & $5(3.9 \%)$ & $6(9.4 \%)$ & 0.185 & \\
\hline ICU stay (days) & $2(1-6)$ & $1.5(1-6)$ & 0.537 & \\
\hline Postoperative RBC transfusion & $11(8.6 \%)$ & 7 (10.9\%) & 0.599 & \\
\hline Postoperative RBC transfused $(U)$ & $4(2-58)$ & $2(2-6)$ & 0.151 & \\
\hline Reoperation & $4(3.1 \%)$ & $0(0.0 \%)$ & 0.303 & \\
\hline Readmission & $0(0.0 \%)$ & $3(4.7 \%)$ & 0.036 & \\
\hline Mortality (in-hospital) & $1(0.8 \%)$ & $0(0.0 \%)$ & 1.000 & \\
\hline Mortality (90-day) & $1(0.8 \%)$ & $0(0.0 \%)$ & 1.000 & \\
\hline Recurrence & $102(79.7 \%)$ & $43(67.2 \%)$ & 0.058 & \\
\hline
\end{tabular}


Table 4 Comparison of intraoperative factors and postoperative short-term and long-term results after PS matching (Continued)

\begin{tabular}{|c|c|c|c|c|}
\hline Variable & Aged $<70$ years $(n=128)$ & Aged $\geq 70$ years $(n=64)$ & $P$ value & Standardized mean difference \\
\hline Intrahepatic recurrence & $70(54.7 \%)$ & $34(53.1 \%)$ & 0.838 & \\
\hline Extrahepatic recurrence & $32(25.0 \%)$ & $9(14.1 \%)$ & 0.081 & \\
\hline Disease-free survival & & & 0.269 & \\
\hline 1-year & $43.5 \%$ & $51.4 \%$ & & \\
\hline 3-year & $21.2 \%$ & $30.3 \%$ & & \\
\hline 5-year & $18.5 \%$ & $24.1 \%$ & & \\
\hline Overall survival & & & 0.401 & \\
\hline 1-year & $89.0 \%$ & $89.1 \%$ & & \\
\hline 3-year & $47.8 \%$ & $57.7 \%$ & & \\
\hline 5-year & $32.7 \%$ & $45.4 \%$ & & \\
\hline Cancer-specific survival & & & 0.163 & \\
\hline 1-year & $89.8 \%$ & $89.1 \%$ & & \\
\hline 3-year & $48.2 \%$ & $61.1 \%$ & & \\
\hline 5-year & $32.9 \%$ & $48.0 \%$ & & \\
\hline
\end{tabular}

the EG and the YG in this study, a PSM method was used to probe the effect of age on patient outcomes after hepatectomy. De Blasi et al. [20] also used this method to mitigate potential biases between groups. However, despite the PSM approach, there were still some unmatched parameters between the groups-namely pedicle clamping duration and recurrence treatment protocol-which resulted in some inconclusive analyses. Furthermore, most previous studies have enrolled patients with EHD, which makes accurate definition of DFS challenging. To overcome this disadvantage, we excluded all patients with EHD from analysis in this study.
Before propensity score matching, there were biases in the baseline data between the EG and the YG due to differences in comorbidities and ASA scores. In addition, some perioperative factors-such as the proportion of patients who had $>3$ liver metastases, received more than six cycles of preoperative chemotherapy, received postoperative chemotherapy, or underwent intraoperative RFA-were also significantly different between the YG and EG. Importantly, it has been reported that more than six cycles of preoperative chemotherapy and intraoperative RFA may increase postoperative morbidity; furthermore, having $>3$ liver metastases and receiving

Table 5 Multivariate Cox regression analyses of disease-free survival, overall survival, and cancer-specific survival after PS matching

\begin{tabular}{|c|c|c|c|}
\hline Variable & Relative ratio & 95\% Confidence interval & $P$ value \\
\hline \multicolumn{4}{|l|}{ Disease-free survival } \\
\hline Age ( $\geq 70$ years old) & 0.860 & $0.602-1.230$ & 0.409 \\
\hline RAS/BRAF mutation & 1.558 & $1.117-2.174$ & 0.009 \\
\hline Preoperative CEA ( $\geq 20 \mathrm{ng} / \mathrm{ml}$ ) & 1.635 & $1.155-2.314$ & 0.006 \\
\hline Preoperative CRS ( $\geq 3$ ) & 1.637 & $1.142-2.347$ & 0.007 \\
\hline Number of Liver metastases (> 3) & 1.732 & $1.120-2.678$ & 0.014 \\
\hline \multicolumn{4}{|l|}{ Overall survival } \\
\hline Age ( $\geq 70$ years old) & 0.828 & $0.550-1.247$ & 0.367 \\
\hline RAS/BRAF mutation & 1.432 & $0.985-2.081$ & 0.060 \\
\hline Preoperative CEA ( $\geq 20$ ng/ml) & 1.699 & $1.157-2.497$ & 0.007 \\
\hline Preoperative CRS ( $\geq 3$ ) & 1.638 & $1.141-2.352$ & 0.007 \\
\hline \multicolumn{4}{|l|}{ Cancer-specific survival } \\
\hline Age ( $\geq 70$ years old) & 0.699 & $0.455-1.076$ & 0.103 \\
\hline RAS/BRAF mutation & 1.348 & $0.911-1.994$ & 0.136 \\
\hline Preoperative CEA ( $\geq 20$ ng/ml) & 2.008 & $1.358-2.968$ & $<0.001$ \\
\hline Preoperative CRS ( $\geq 3$ ) & 1.548 & $1.069-2.241$ & 0.021 \\
\hline
\end{tabular}


postoperative adjuvant chemotherapy are important prognostic factors for patients with CRLM $[25,26]$. Propensity score matching was used to balance the distribution of these variables between groups in this study. After matching, short-term patient outcomes revealed that the EG had significantly less intraoperative blood loss and a shorter postoperative hospital stay-though a higher readmission rate-compared to the YG. Longterm outcomes demonstrate a slight, but nonstatistically significant improvement, in 5-year DFS, OS, and CSS for the EG compared with the YG.

For modern treatment of CRLM, routine testing of RAS/BRAF mutation status has been recommended since 2014 as it has been confirmed to be a negative prognostic factor for CRLM patients [27]. It has been reported that positive RAS/BRAF mutation status is associated with shorter DFS and OS and narrower margin widths after hepatectomy compared to wild-type RAS/ BRAF [28-30]. However, RAS/BRAF mutation status has never been considered in previous retrospective studies, as much of the data precedes standard testing for RAS/BRAF status. In this study, all tumor tissue samples from the considered patients were retrieved from the pathology department and tested for RAS/ BRAF mutation status, which was matched between the two groups after PSM.

Primary tumor sidedness has also been emphasized in recent years in recognition of the fact that the side of origin plays a role in tumor behavior and progression. It was reported that tumors originating on the right were more frequently associated with female patients, the elderly, high grade (poor differentiation), BRAF mutations, the enhanced $\mathrm{CpG}$ island methylator phenotype, high microsatellite instability, and high expression of consensus molecular subtypes 1 and 3 compared with left-side origin tumors [31]. These characteristics negatively affect anti-EGFR treatment and the prognosis of patients with right-sided tumors [32]. Therefore, we included primary tumor sidedness in our PSM model to balance the possible bias associated with this disease feature.

The CRS was proposed in 1999 by Fong et al. [33] as a prognostic indicator composed of five preoperative variables: preoperative CEA $>200 \mathrm{ng} / \mathrm{mL}$, primary positive lymph nodes, an interval of $<12$ months between diagnosis of the primary tumor and liver metastasis, presence of multiple liver metastases, and maximal diameter of liver metastases $>5 \mathrm{~cm}$. It has been shown that increased CRS is associated with an increased risk of postoperative recurrence and death [34]. Thus, it was important to include CRS in our PSM analysis to appropriately examine the effect of age on survival.

This study revealed no significant difference in either general or surgical postoperative complications between the EG and the YG. This result aligns with previous studies and suggests that surgery for elderly patients is as safe as it would be for younger patients with the same ASA score and comorbidities [20,35]. This holds even when simultaneous resection of primary and liver tumors or major hepatectomy is performed. Interestingly, compared to the YG, the EG was found to have significantly less intraoperative blood loss $(175 \mathrm{~mL}$ vs. $200 \mathrm{~mL}, p=$ $0.046)$ and a shorter median postoperative hospital stay (8 days vs. 11 days, $p=0.020$ ), which might reflect that appropriately selected elderly recover promptly from surgery. However, the EG group did have a significantly higher readmission rate than the YG $(4.7 \%$ vs. $0 \%, p=$ 0.036). Although the median intraoperative blood loss of the YG is higher than that of the EG, an absolute difference of $25 \mathrm{~mL}$ is of little clinical significance. The significance of the difference between groups may result from the relatively small sample size of this study, considering the $p$ value approaching 0.05 . With a larger sample size and improved matching between the groups with regard to perioperative details-such as preoperative chemotherapy, major hepatectomy, simultaneous resection, and Pringle clamping time-the intraoperative blood loss will likely be comparable between the EG and the YG.

The three patients from the EG who were readmitted to the hospital were all 71 years of age. The first patient was diagnosed with synchronous descending colon cancer with liver metastases and received simultaneous resection of the primary tumor and liver metastases. The patient was readmitted due to incisional infection 2 months after discharge. The second readmitted patient was diagnosed with bilobar liver metastases after resection of sigmoid colon cancer. He was readmitted to the hospital 1 month after liver resection due to a fever of $38.3^{\circ} \mathrm{C}$. Laboratory tests showed a normal white blood cell count, and radiological examinations revealed no signs of abdominal or thoracic collection. The third readmitted patient was diagnosed with multiple liver metastases after resection of ascending colon cancer. He received hepatectomy and intraoperative RFA for multiple tumors. He was readmitted to the hospital 2 months after operation due to fever of $38.5^{\circ} \mathrm{C}$. The white blood cell count was marginally elevated, and ultrasound showed a small thoracic collection without abdominal fluid. In brief, although three patients from the EG were readmitted to the hospital, only one of them experienced an unequivocal surgical complication (incisional infection), which classified as a minor complication. None of the readmitted patients experienced systemic complications related to old age. When the length of hospital stay after readmission was added to the length of the postoperative hospital stay, the median total length of postoperative hospital stay was still significantly longer in the YG than in the EG (11 days vs. 8 days, $p=0.024$ ). As such, we do not believe that the EG's length of stay advantage compared to the YG was offset by the higher readmission rate. 
With regard to the median length of postoperative stay being shorter in EG patients compared to YG patients, we found that the proportions of major hepatectomy and grade III or higher postoperative complications were much higher in the YG than the EG (18.8\% vs. $12.5 \%$ and $56.8 \%$ vs. $29.4 \%$, respectively), although this was not statistically significant. Given the relatively small sample size of our study, the significantly shorter postoperative length of stay in the EG may result from these differences. Thus, this result should be clarified in larger studies in the future. Regarding long-term patient outcomes, surprisingly, the 5-year DFS, OS, and CSS rates were increased by $5.6 \%, 12.7 \%$, and $15.1 \%$, respectively, for the EG compared to the YG, although these differences were not statistically significant. Importantly, it was shown that the OS and CSS of the YG were longer than those of the EG prior to PSM. This discrepancy may arise due to the following factors. First, some research [36, 37] has shown that the malignancy of tumors in the elderly population may be reduced; thus, the potential for tumor growth and metastasis may be decreased in the elderly as well. Second, some factors such as RAS/BRAF mutation status, primary tumor sidedness, and history of preoperative chemotherapy were included in our study and balanced between the EG and YG by PSM. These important prognostic factors were lacking in previously published studies, which may contribute to the difference in OS identified in our study. Notably, due to the relatively small sample size of this study, this conclusion should be confirmed by high-quality studies with a larger sample size in the future.

This study has some limitations. First, similar to other studies, this study had a relatively small sample size. After eliminating patients with EHD and unmatched patients, there were only 64 patients in the EG and 128 patients in the matched YG considered for analysis, which may impact the representativeness and robustness of the results. As such, the results of this study should be confirmed by high-quality studies with larger sample sizes in the future. Another limitation is the retrospective nature of this study, so the conclusion should be evaluated by large prospective controlled trials in future work. The third limitation is the loss of information regarding the response to preoperative chemotherapy as a referral center, which has been shown in previous work to be a pivotal prognostic factor for CRLM patients undergoing hepatectomy $[34,38]$. Finally, the fourth limitation was that the chemotherapy regimes had changed during so long study interval. Actually, preoperative and postoperative chemotherapy backbone drugs of the younger group were not significantly different from those of the elderly group as shown in Table 3 . However, the detailed chemotherapy regimes were very difficult to be balanced between the two groups.

\section{Conclusions}

For appropriately identified elderly patients with colorectal liver-limited metastases, hepatectomy is safe and effective. In this study, we found that there was no increase in postoperative morbidities and mortality compared with matched younger patients. Importantly, elderly patients may benefit from longer DFS, OS, and CSS after hepatectomy; thus, this procedure should be performed for elderly patients who are in the same performance status with adequate cardiopulmonary reserve as the younger patients, especially for those patients with favorable biological behavior.

\section{Abbreviations}

PSM: Propensity score matching; YG: Younger group; EG: Elderly group; OS: Overall survival; DFS: Disease-free survival; CSS: Cancer-specific survival; RAS: Rat sarcoma virus proto-oncogene; BRAF: B-Raf proto-oncogene; EHD: Extrahepatic diseases; ASA: American Society of Anesthesiologists; CRS: Clinical risk score; CEA: Carcinoembryonic antigen; CA19-

9: Carbohydrate antigen 19-9; RBC: Red blood cell; RFA: Radiofrequency ablation; CRLM: Colorectal liver metastases

\section{Acknowledgements}

We would like to thank Mr. Wen-Wu He and Miss Cathy He for their efforts in revising the manuscript.

\section{Authors' contributions}

KM J designed the study, analyzed the data, and wrote the manuscript. K W, Q B, and HW W participated in the data collection. BC X participated in the study design and revised the manuscript. All authors read and approved the final manuscript.

\section{Funding}

This work was supported by the National Natural Science Foundation of China (grant No. 81874143 and No. 31971192), National Key Basic Research Development Program of China (grant No. 2014CBA02001), and the Natural Science Foundation of Beijing Municipality (grant No. 7192035).

\section{Availability of data and materials}

The data that support the findings of this study are available on request from the corresponding author. The data are not publicly available due to privacy or ethical restrictions.

\section{Ethics approval and consent to participate}

The Ethics Committee of Beijing Cancer Hospital approved the study protocol (reference No. 2015KT32), which was performed in compliance with the Helsinki Declaration.

\section{Consent for publication}

We just extracted data and did not involve the private information of patients.

\section{Competing interests}

There are no conflicts of interest to declare.

Received: 4 August 2020 Accepted: 15 October 2020

Published online: 24 October 2020

\section{References}

1. Bray F, Ferlay J, Soerjomataram I, et al. Global cancer statistics 2018: GLOBOCAN estimates of incidence and mortality worldwide for 36 cancers in 185 countries. CA Cancer J Clin. 2018;68(6):394-424.

2. Leporrier J, Maurel J, Chiche L, et al. A population-based study of the incidence, management and prognosis of hepatic metastases from colorectal cancer. Br J Surg. 2006;93(4):465-74.

3. van der Pool AE, Damhuis RA, ljzermans JN, et al. Trends in incidence, treatment and survival of patients with stage IV colorectal cancer: a population-based series. Colorectal Dis. 2012;14(1):56-61. 
4. Morris EJ, Forman D, Thomas JD, et al. Surgical management and outcomes of colorectal cancer liver metastases. Br J Surg. 2010;97(7):1110-8.

5. de Jong MC, Pulitano C, Ribero D, et al. Rates and patterns of recurrence following curative intent surgery for colorectal liver metastasis: an international multi-institutional analysis of 1669 patients. Ann Surg. 2009; 250(3):440-8.

6. Rees M, Tekkis PP, Welsh FK, et al. Evaluation of long-term survival after hepatic resection for metastatic colorectal cancer: a multifactorial model of 929 patients. Ann Surg. 2008;247(1):125-35.

7. Wei AC, Greig PD, Grant D, et al. Survival after hepatic resection for colorectal metastases: a 10-year experience. Ann Surg Oncol. 2006;13(5):668-76.

8. Choti MA, Sitzmann JV, Tiburi MF, et al. Trends in long-term survival following liver resection for hepatic colorectal metastases. Ann Surg. 2002; 235(6):759-66.

9. Mavros MN, Hyder O, Pulitano C, et al. Survival of patients operated for colorectal liver metastases and concomitant extra-hepatic disease: external validation of a prognostic model. J Surg Oncol. 2013;107(5):481-5.

10. Soreide K, Wijnhoven BP. Surgery for an ageing population. Br J Surg. 2016; 103(2):e7-9.

11. Adam R, Frilling A, Elias D, et al. Liver resection of colorectal metastases in elderly patients. Br J Surg. 2010;97(3):366-76.

12. Mann CD, Neal CP, Pattenden CJ, et al. Major resection of hepatic colorectal liver metastases in elderly patients - an aggressive approach is justified. Eur J Surg Oncol. 2008;34(4):428-32.

13. Bockhorn M, Sotiropoulos GC, Sgourakis G, et al. Major liver resections in the elderly-is an aggressive approach justified? Int J Colorectal Dis. 2009;24(1):83-6.

14. Kulik U, Framke T, Grosshennig A, et al. Liver resection of colorectal liver metastases in elderly patients. World J Surg. 2011;35(9):2063-72.

15. Nachmany I, Pencovich N, Zohar N, et al. Resection of colorectal liver metastases in the elderly-is it justified? J Surg Oncol. 2016;113(5):485-8.

16. Tufo A, Dunne DF, Manu N, et al. Changing outlook for colorectal liver metastasis resection in the elderly. Eur J Surg Oncol. 2019;45(4):635-43.

17. Cook EJ, Welsh FK, Chandrakumaran $K$, et al. Resection of colorectal liver metastases in the elderly: does age matter? Colorectal Dis. 2012;14(10):1210-6.

18. Booth CM, Nanji S, Wei X, et al. Management and outcome of colorectal cancer liver metastases in elderly patients: a population-based study. JAMA Oncol. 2015;1(8):1111-9.

19. Kumari S, Semira C, Lee M, et al. Resection of colorectal cancer liver metastases in older patients. ANZ J Surg. 2020;90(5):796-801.

20. De Blasi V, Memeo R, Adam R, et al. Major hepatectomy for colorectal liver metastases in patients aged over 80: a propensity score matching analysis. Dig Surg. 2018;35(4):333-41.

21. Ingravallo F, Cerquetti I, Vignatelli L, et al. Medico-legal assessment of personal damage in older people: report from a multidisciplinary consensus conference. Int J Legal Med. 2020. https://doi.org/10.1007/s00414-020-02368-z.

22. van Tuil T, Dhaif AA, Te Riele WW, et al. Systematic review and meta-analysis of liver resection for colorectal metastases in elderly patients. Dig Surg. 2019;36(2):111-23.

23. Zacharias T, Jaeck D, Oussoultzoglou $E$, et al. First and repeat resection of colorectal liver metastases in elderly patients. Ann Surg. 2004;240(5):858-65.

24. Nagano $Y$, Nojiri $K$, Matsuo $K$, et al. The impact of advanced age on hepatic resection of colorectal liver metastases. J Am Coll Surg. 2005;201(4):511-6.

25. Mima K, Beppu T, Chikamoto A, et al. Hepatic resection combined with radiofrequency ablation for initially unresectable colorectal liver metastases after effective chemotherapy is a safe procedure with a low incidence of local recurrence. Int J Clin Oncol. 2013;18(5):847-55.

26. Nakano $\mathrm{H}$, Oussoultzoglou E, Rosso $\mathrm{E}$, et al. Sinusoidal injury increases morbidity after major hepatectomy in patients with colorectal liver metastases receiving preoperative chemotherapy. Ann Surg. 2008;247(1):118-24.

27. Van Cutsem E, Cervantes A, Nordlinger B, et al. Metastatic colorectal cancer: ESMO Clinical Practice Guidelines for diagnosis, treatment and follow-up. Ann Oncol. 2014;25(Suppl 3):iii1-9.

28. Van Cutsem E, Lenz HJ, Kohne CH, et al. Fluorouracil, leucovorin, and irinotecan plus cetuximab treatment and RAS mutations in colorectal cancer. J Clin Oncol. 2015;33(7):692-700.

29. Brudvik KW, Mise Y, Chung MH, et al. RAS mutation predicts positive resection margins and narrower resection margins in patients undergoing resection of colorectal liver metastases. Ann Surg Oncol. 2016;23(8):2635-43.

30. Kadowaki S, Kakuta M, Takahashi S, et al. Prognostic value of KRAS and BRAF mutations in curatively resected colorectal cancer. World J Gastroenterol. 2015;21(4):1275-83.
31. Lee MS, Menter DG, Kopetz S. Right versus left colon cancer biology: integrating the consensus molecular subtypes. J Natl Compr Canc Netw. 2017;15(3):411-9.

32. Huemer $F$, Thaler J, Piringer $G$, et al. Sidedness and TP53 mutations impact OS in anti-EGFR but not anti-VEGF treated mCRC - an analysis of the KRAS registry of the AGMT (Arbeitsgemeinschaft Medikamentose Tumortherapie). BMC Cancer. 2018;18(1):11.

33. Fong $Y$, Fortner J, Sun RL, et al. Clinical score for predicting recurrence after hepatic resection for metastatic colorectal cancer: analysis of 1001 consecutive cases. Ann Surg. 1999;230(3):309-18 discussion 318-21.

34. Xu D, Liu XF, Yan XL, et al. Survival prediction in patients with resectable colorectal liver metastases: clinical risk scores and tumor response to chemotherapy. Oncol Lett. 2017;14(6):8051-9.

35. Nomi T, Fuks D, Kawaguchi Y, et al. Laparoscopic major hepatectomy for colorectal liver metastases in elderly patients: a single-center, case-matched study. Surg Endosc. 2015;29(6):1368-75.

36. Itzhaki O, Kaptzan T, Skutelsky E, et al. Age-adjusted antitumoral therapy based on the demonstration of increased apoptosis as a mechanism underlying the reduced malignancy of tumors in the aged. Biochim Biophys Acta. 2004;1688(2):145-59.

37. Han Z, Brown JR, Niederkorn JY. Growth and metastasis of intraocular tumors in aged mice. Invest Ophthalmol Vis Sci. 2016;57(6):2366-76.

38. Wang K, Liu W, Yan XL, et al. Long-term postoperative survival prediction in patients with colorectal liver metastasis. Oncotarget. 2017;8(45):79927-34.

\section{Publisher's Note}

Springer Nature remains neutral with regard to jurisdictional claims in published maps and institutional affiliations.

Ready to submit your research? Choose BMC and benefit from:

- fast, convenient online submission

- thorough peer review by experienced researchers in your field

- rapid publication on acceptance

- support for research data, including large and complex data types

- gold Open Access which fosters wider collaboration and increased citations

- maximum visibility for your research: over $100 \mathrm{M}$ website views per year

At BMC, research is always in progress.

Learn more biomedcentral.com/submissions 